\title{
Influence of variation of the distance between centers of the sliding speed gear drives: Application to the crowns of sugar cane mills
}

\author{
Dr. Jorge Laureano Moya Rodríguez ${ }^{1}$, Dr. Rafael Goytisolo Espinosa ${ }^{2}$, Dra. Maida Bárbara Reyes Rodríguez ${ }^{3}$ \\ 1,2,3Facultad de Ingeniería Mecánica, Universidad Central “Marta Abreu” de Las Villas. Cuba. (jorgemr@uclv.edu.cu)
}

\begin{abstract}
In gear transmissions there is a great wear, because there is a sliding velocity between tooth. This sliding in spite of be inevitable has great repercussion over Tooth wear. In gears of sugar mill rollers distance between centers changes during operation and wear is bigger. For this purpose full involute gears are used and sliding increases. Expressions for calculating sliding velocities in such gears are shown in this article.
\end{abstract}

Keywords: Transmitters of Engrenagens, sugar cane, Application to the crowns.

\section{Influencia de la variación de la distancia entre centros en la velocidad de deslizamiento de las transmisiones por engranajes. Aplicación a las coronas de molinos de caña de azucar}

\section{RESUMEN}

En las transmisiones por engranajes de perfil evolvente se produce un alto deslizamiento entre los dientes, ya que existe una componente de la velocidad a lo largo del diente. Este deslizamiento a pesar de ser inevitable, tiene repercusiones considerables en el desgaste del perfil. Existen numerosas aplicaciones industriales donde las transmisiones por engranajes trabajan con variación de la distancia entre centras de operación, tales como los molinos de caña de azúcar, trenes de laminado, etc. En estos casos se usan engranajes de evolvente alargada, siendo aun mayor el deslizamiento de un perfil sobre el otro, al existir una nueva componente de la velocidad producto del movimiento de aproximación y separación de las ruedas dentadas de acuerdo a la abertura del molino. En el trabajo se hace un análisis de las expresiones para el cálculo de las velocidades de deslizamiento de este tipo de transmisiones; las cuales no aparecen en la literatura tradicional de transmisiones por engranajes. Se muestran programas de computación para el cálculo de estas velocidades.

Palavras-Chave: Transmisiones por engranajes, caña de azucar, Aplicación a las coronas de molinos.

\section{INTRODUCCÍON}

El desgaste es una de las fallas fundamentales de las transmisiones por engranajes que trabajan con condiciones deficientes de lubricación [1][2]. Esta situación se ve agravada aun más si los engranajes trabajan además con variación de la distancia entre centros durante la explotación. Tal es el caso de las coronas de molinos de caña de azúcar, donde esta es una de las principales causas de deterioro. En Cuba se utiliza una amplia gama de coronas de molinos con diferentes parámetros geométricoa (número de dientes, módulo, etc.). Estos perfiles se designan por letras que van desde la $\mathrm{A}$ hasta la $\mathrm{P}$ en orden decendente de tamaño.

En los engranajes de evolvente que trabajan con distancia entre centros constante este fenómeno ha sido estudiado profundamente por numerosos autores, no obstante en engranajes de perfil especial que trabajan con una variación continua de la distancia entre centros no ha sido estudiado a profundidad, ya que estos casos solamente se presentan en aplicaciones muy específicas.

El desgaste de una transmisión dentada depende de muchos factores, pero no cabe lugar a dudas de que el deslizamiento juega 
n papel decisivo en el mismo [3][4]. A continuación se desarrolla todo un procedimiento matemático para calcular el deslizamiento en transmisiones que trabajan con variación de la distancia entre centros, especificamente las coronas de molinos cañeros, donde el desgaste es una limitante en la capacidad de carga de las mismas.

\section{DESARROLLO}

En la figura 1 se muestra el esquema de dos ruedas engranadas, y se analizan las velocidades que se producen en el punto $\mathrm{K}$ del contacto.

Como se aprecia de la misma hay una diferencia de velocidades en el punto de contacto, produciéndose una velocidad de deslizamiento en el perfil del diente. Esta velocidad de deslizamiento se calcula por el procedimiento que se describe a continuación [5][6]:

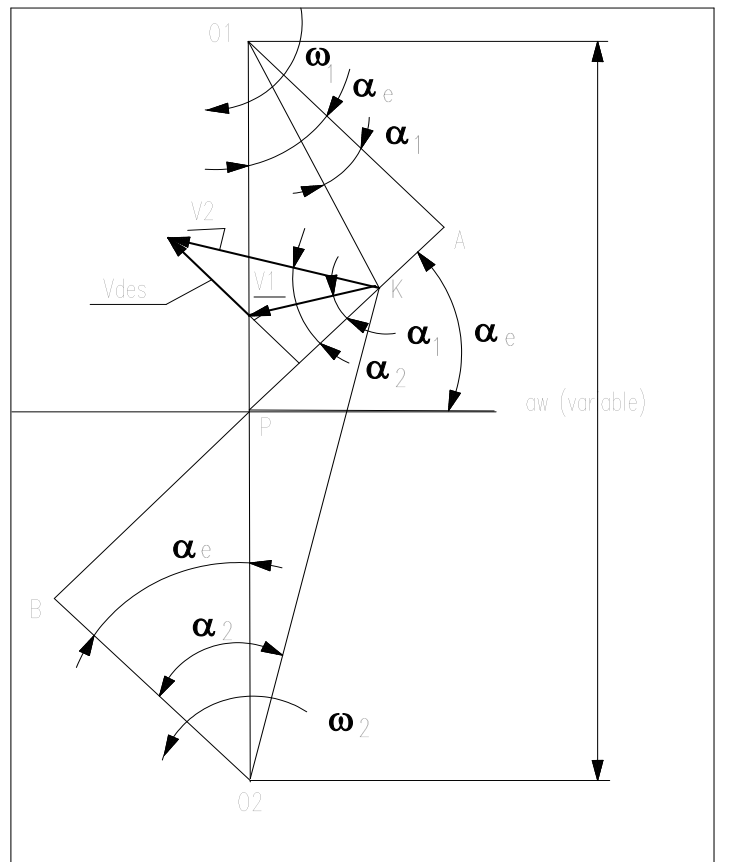

Figura. 1: Esquema de dos ruedas engranadas.

$$
\begin{gathered}
V_{d e s}=V_{2} \cdot \sin \alpha_{2}-V_{1} \cdot \sin \alpha_{1} \\
V_{d e s}=\omega_{2} o_{2} k \cdot \sin \alpha_{2}-\omega_{1} o_{1} k \sin \alpha_{1}
\end{gathered}
$$

En los engranajes de los molinos de caña de azúcar (coronas de molinos):

$$
\omega_{2}=\omega_{1}=\omega
$$

por tanto:

$$
V_{d e s}=\omega \cdot(B K-A K)=\omega \cdot(A B-A K-A K)=\omega \cdot(A B-2 A K)(4)
$$

De donde:

$$
V_{d e s}=\omega \cdot\left(2 \sqrt{\left(\frac{a_{w}}{2}\right)^{2}-\left(\frac{d_{o}}{2}\right)^{2}}-d_{o} \cdot \tan \alpha_{1}\right)
$$

Teniendo en cuenta que:

$$
d_{o}=m \cdot Z \cdot \cos \alpha_{p}
$$

donde:

$\alpha_{\mathrm{p}}$ - Ángulo del perfil del diente

m- Módulo

Z- Número de dientes de la corona.

se llega a la expresión:

$$
V_{d e s}=\omega \cdot\left[\sqrt{a_{w}^{2}-\left(m \cdot Z \cdot \cos \alpha_{p}\right)^{2}}-m \cdot Z \cdot \cos \alpha_{p} \cdot \tan \alpha_{1}\right]
$$

En la expresión anterior

$\omega$ - Velocidad angular a la que gira la corona

$\mathrm{a}_{\mathrm{W}^{-}}$Distancia entre centros (variable)

$\alpha_{1}$ - Ángulo variable para un punto intermedio del contacto.

Para el caso particular del inicio del contacto, donde el deslizamiento es más crítico, la expresión anterior toma la siguiente forma:

$$
\begin{gathered}
V_{d e s}=\omega \cdot(B K-A K)=\omega \cdot[B K-(A B-B K)]=\omega \cdot(2 B K-A B) \\
A B=\sqrt{a_{w}^{2}-d_{o}^{2}} \\
B K=\sqrt{\left(\frac{d_{a}}{2}\right)^{2}-\left(\frac{d_{o}}{2}\right)^{2}} \\
V_{d e s}=\omega \cdot\left(\sqrt{d_{a}^{2}-d_{o}^{2}}-\sqrt{a_{w}^{2}-d_{o}^{2}}\right)
\end{gathered}
$$

Para conocer como varia la velocidad de deslizamiento en función de los parámetros del dentado y de operación se confeccionó un programa de computación. Se analizó la influencia de tres parámetros básicos: el ángulo del perfil, el número de dientes, y la distancia entre centros. Los resultados de la ejecución de los programas se muestran en las figuras $2,3, \mathrm{y} 4$. 


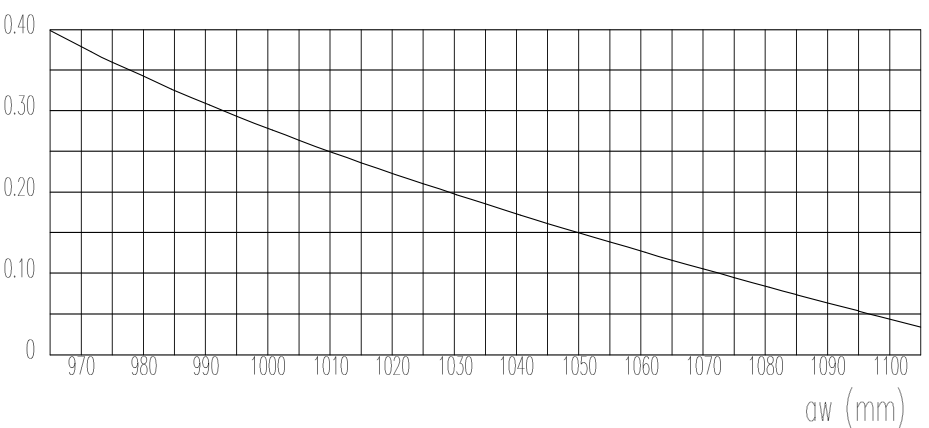

Figura 2: Variación de la velocidad de deslizamiento en función de la distancia entre centros para el perfil A en el inicio del contacto de los dientes.

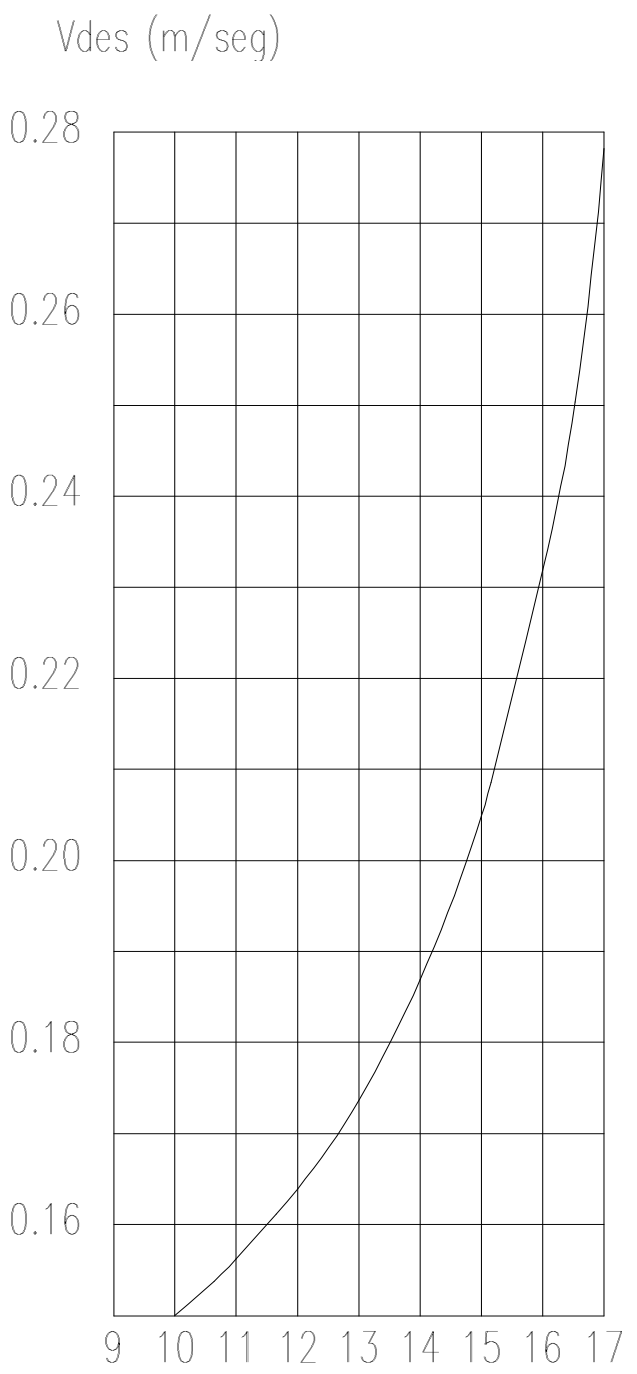

Figura 3: Variación de la velocidad de deslizamiento en función del número de dientes para coronas tipo A en el punto de inicio del contacto.

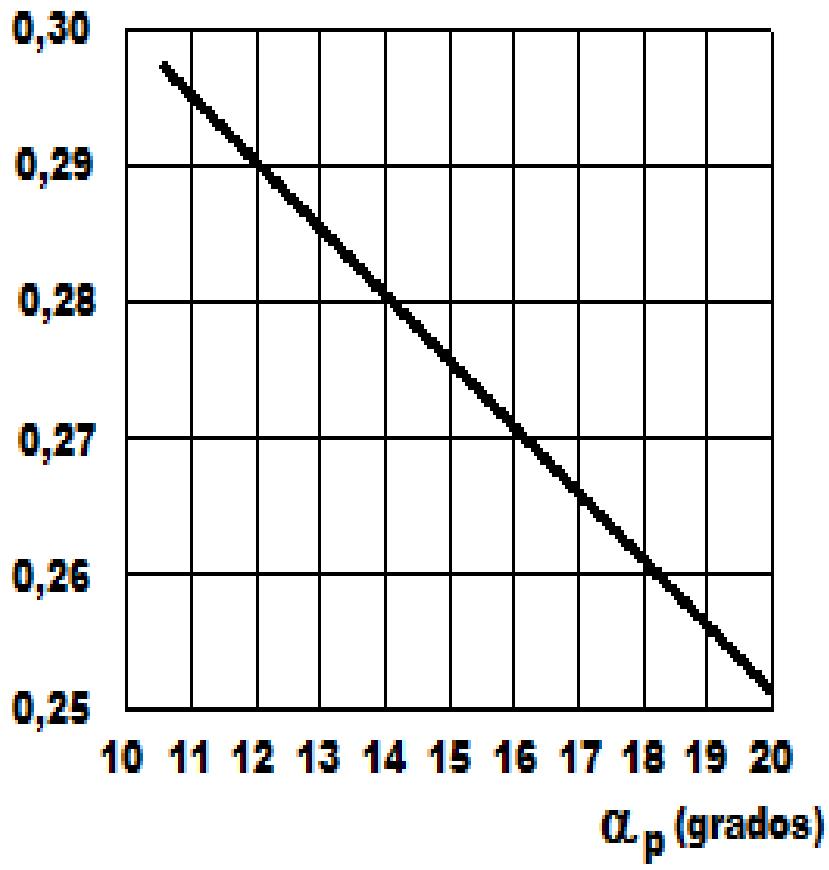

Figura. 4: Variación de la velocidad de deslizamiento en función del ángulo del perfil para coronas tipo A en el inicio del contacto entre los dientes.

Para poder establecer un criterio comparativo entre el deslizamiento en dos coronas, y por ende del desgaste en las mismas, se estableció el concepto de índice de desgaste, el cual se puede calcular por la siguiente expresión:

$$
I_{d e s}=\frac{V_{d e s n}}{V_{d e s p}}
$$

Donde:

$V_{\text {desn }}{ }^{-}$Velocidad de deslizamiento del perfil del diente de la corona que se desea probar.

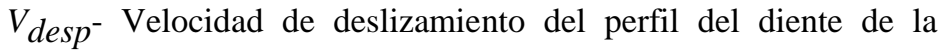
corona que se desea usar como patrón.

En las figuras 5 y 6 se muestra como varia el índice de desgaste en función de los parámetros del dentado con relación a las coronas de perfil A conservando las dimensiones exteriores del mismo. 


\section{Ides}

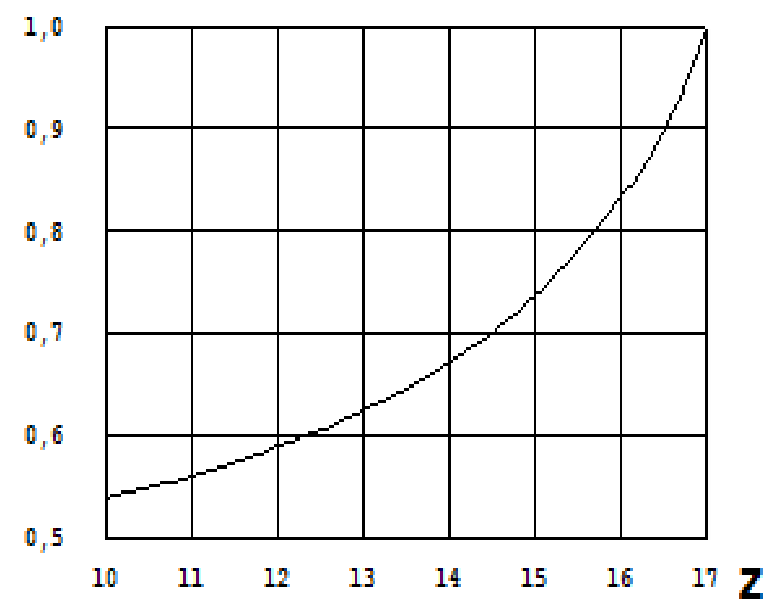

Figura. 5: Variación del índice de deslizamiento en función del número de dientes con respecto a las coronas tipo $\mathrm{A}$.

Un aspecto importante dentro de la geometría de los dientes es el radio de los diferentes arcos que conforman el perfil, ya que a medida que este valor sea mayor, serán menores las tensiones de contacto.

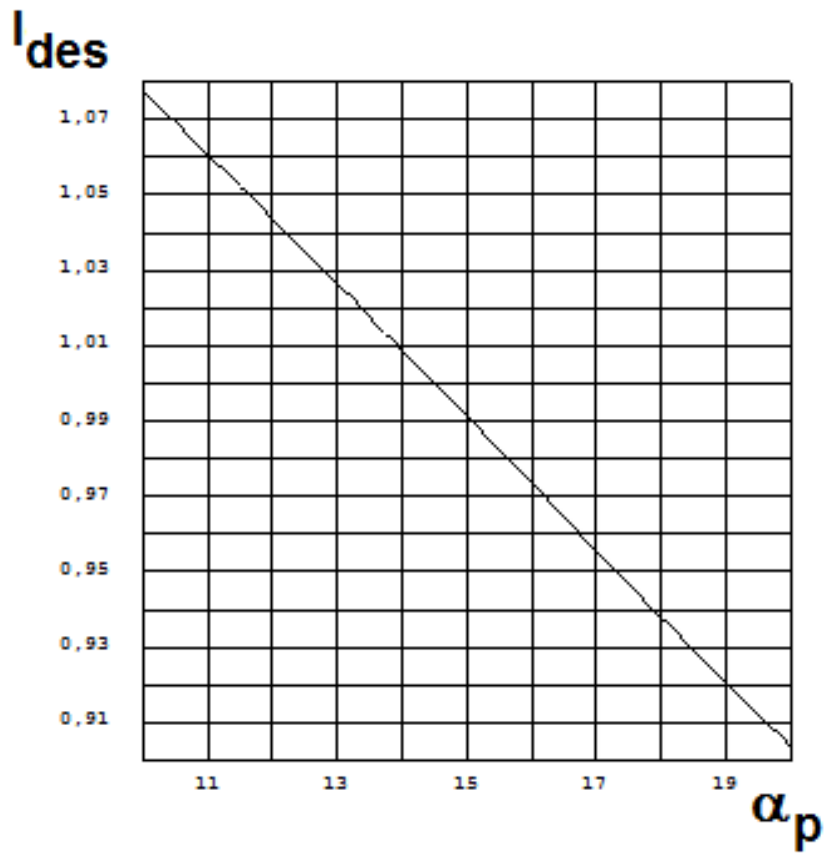

Figura. 6: Variación del índice de deslizamiento en función del ángulo del perfil con respecto a las coronas tipo A para una distancia de operación de $1000 \mathrm{~mm}$.

\section{CONCLUSIONES}

La expresión para el cálculo de la velocidad de deslizamiento en las transmisiones que trabajan con variación de la distancia entre centros es parecida a la de los engranajes convencionales, sólo que en la misma hay un parámetro que es variable (la distancia entre centros). En ocasiones los valores de velocidad de deslizamiento calculados para engranajes que operan con variación de la distancia entre centros es mayor que en los engranajes convencionales, en otras es menor. A medida que la distancia entre centros disminuye se está en presencia de mayores valores de la velocidad de deslizamiento.

A medida que aumenta el número de dientes la velocidad de deslizamiento y el índice de deslizamiento aumentan.

A medida que aumenta el ángulo del perfil la velocidad de deslizamiento y el índice de deslizamiento disminuye.

Teniendo en cuenta las consideraciones anteriores el perfil del diente es determinante cuando las transmisiones por engranajes trabajan con variación de la distancia entre centros, con vistas a que el valor de la velocidad de deslizamiento sea lo menor posible.

\section{REFERENCIAS BIBLIOGRAFICAS}

[1] Inoue, S., Ikejo, K., Nagamura, K., Seyama, N., \& Nakagawa, S. (2013). A Prediction Method of Wear on Tooth Surface for Spur Gears. Paper presented at the ASME 2013 International Design Engineering Technical Conferences and Computers and Information in Engineering Conference.

[2] Venkatesh, B., Vattikuti, S. P., \& Reddy, V. M. (2014). Investigate the Combined Effect of gear ratio, helix angle, face width and module on beam strength and Wear tooth load of Steel alloy Helical Gear.

[3] Jiang, H., Shao, Y., \& Mechefske, C. K. (2014). Dynamic characteristics of helical gears under sliding friction with spalling defect. Engineering Failure Analysis, 39, 92-107.

[4] Masjedi, M., \& Khonsari, M. (2015). On the prediction of steady-state wear rate in spur gears. Wear, 342, 234-243.

[5] Onishchenko, V. (2015). Investigation of tooth wears from scuffing of heavy duty machine spur gears. Mechanism and Machine Theory, 83, 38-55.

[6] Yenti, C., Phongsupasamit, S., \& Ratanasumawong, C. (2012). Analytical and Experimental Investigation of Parameters Affecting Sliding Loss in a Spur Gear Pair. Engineering Journal, 17(1), 79-94. 